\title{
Swallowing impairment in neurologic disorders: the role of videofluorographic swallowing study
}

\author{
Giuseppe Lo Re ${ }^{\mathrm{E}}$, Maria Chiara Terranova ${ }^{\mathrm{B}}$, Federica Vernuccio ${ }^{\mathrm{F}}$, Claudia Calafiore ${ }^{\mathrm{B}}$, Dario Picone ${ }^{\mathrm{F}}$, \\ Chiara Tudisca ${ }^{\mathrm{E}}$, Sergio Salerno ${ }^{\mathrm{A}}$, Roberto Lagalla ${ }^{\mathrm{A}}$ \\ Section of Radiological Sciences, DIBMIMEF, University Hospital Policlinico, University of Palermo, Palermo, Italy
}

\section{Abstract}

Patients with neurologic diseases almost inevitably develop various degrees of swallowing disorders during their life. Dysphagia is one of the main negative prognostic factors in this class of patients, leading to severe morbidity (i.e. aspiration pneumonia, dehydration, malnutrition, and life quality deterioration) and to a noticeable increase in public health spending.

Videofluorographic swallowing study is considered the gold standard technique for swallowing impairment assessment. It is aimed at early identification of the risk of aspiration, definition of the kind and grade of dysphagia, and an indication to suspend oral nutrition and adopt other feeding strategies, and define when the patient is able to return to physiological nutrition.

Every radiologist should be familiar with the main videofluorographic swallowing features in neurological patients, not only because early diagnosis of deglutition disorders widely improves their prognosis, but also because customising feeding strategies has a great impact on patients' quality of life.

Key words: videofluorography, swallowing, neurologic diseases, dysphagia.

\section{Introduction}

Dysphagia is defined as difficulty or discomfort during bolus progression along the upper gastrointestinal tract, most commonly due to anomalies in the swallowing process, representing one of the main negative prognostic factors in patients with neurologic diseases [1].

Swallowing results from a complex sensorimotor integration, which is the key point for the correct coordination and interconnection between the 3-4 phases of swallowing, which are: oral (preparatory and propulsive), pharyngeal, and oesophageal phases [1,2].

Radiographic evaluation of swallowing through videofluorographic swallowing study (VFSS) is often the first step for evaluating dysphagia in neurologic patients, to- gether with clinical examination, in order to assess the severity of dysphagia and to formulate feeding and nutritional approaches, along with overall patient management [1-7]. The role of this technique is to evaluate the patient and how he/she transfers the bolus from mouth to oesophagus.

\section{Videofluorographic swallowing study}

VFSS provides both structural and functional information on the swallowing process, and it is performed with contrast media (CM) administration, even if a preliminary non-contrast examination can be performed, in order to evaluate tongue and soft palate movements and symmetry of vocal folds closure $[5,6]$.

Correspondence address:

Prof. Sergio Salerno, Section of Radiological Sciences, DIBMIMEF, University Hospital Policlinico, University of Palermo, Via del Vespro 127, Palermo, 90127, Italy, e-mail: sergio.salerno@unipa.it

Authors' contribution:

A Study design · B Data collection · C Statistical analysis · D Data interpretation · E Manuscript preparation · F Literature search · G Funds collection 
Although a detailed description of the technique goes beyond the purpose of this review, some key-points of the VFSS are described in order to explain abnormal findings in neurologic patients.

Rheological characteristics and consistency of CM bolus vary widely - liquid CM, semi-liquid CM, toasted bread or even marshmallows dipped in $\mathrm{CM}$ - aiming to assess which food consistency is difficult to swallow, thus guiding nutritional management [1,6-8].

Usually barium solutions are the most commonly used CM in VFSS, but when aspiration or any kind of upper gastro-intestinal tract perforation is suspected barium must be avoided, and water-soluble CM is preferable.

The endpoints of the oral phase for swallowing effectiveness are labial competence, tongue efficacy in pushing the bolus towards the pharynx, palatoglossal closure, and complete oral cavity emptying [6].

In pharyngeal stage the following parameters are evaluated: the palate-pharyngeal closure - which avoids bolus regurgitation in the rhino-pharynx - and the triggering of the swallowing reflex, hyoid and laryngeal elevation, epiglottal tilting, and complete clearing of the pharyngeal cavity $[1,3,5,6-8]$.

In every dysphagic patient, the evaluation of bolus transit until lower oesophageal sphincter is suggested, in standing and supine or prone position, in order to detect oesophageal clearing and peristalsis and lower oesophageal sphincter continence [1,6-8].

In patients with neurological diseases, modifying head posture while performing the exam is strongly recommended, not only to disclose eventual undetected swallowing disorders, but also to identify more appropriate posture that can simplify or evoke airway protection mechanisms [1,6-8].

This latter is the main point to be assessed during VFSS examination.

On frontal view epiglottal tilting symmetry is evaluated. Lateral view acquisition offers the possibility to detect the presence of bolus laryngeal leakage and to distinguish between penetration (defined when the CM reaches laryngeal vestibule, above the vocal folds) and aspiration (when the CM progresses into the lower airways) and temporal interconnection between the deglutition phases: pre-, intra-, and post-deglutitive aspiration $[1,2]$.

The presence of a clinician and a nutritional specialist or a speech therapist during examination is strongly recommended, in order to tailor VFSS to the patient's characteristics and to identify the more appropriate CM consistency and to test the effectiveness of compensative manoeuvres and postural strategies. Moreover, their role in detecting potential swallowing impairments and in training the patient to perform compensatory manoeuvres is crucial, especially in the case of cognitive impairment and to reduce eventual exam-induced stress and anxiety [9].

In this review we systematically describe typical findings on VFSS in patients with neurologic diseases and sec- ondary dysphagia $[1,2]$. Because radiologic evaluation of oral-pharyngeal function is an extension of the neurologic function of specific cranial nerves, VFSS is crucial for assessing swallowing dysfunctions, estimating the severity of the disease, and planning appropriate treatment $[1,4]$.

\section{Amyotrophic lateral sclerosis}

Amyotrophic lateral sclerosis (ALS) is a progressive neurodegenerative disease that affects cortical and spinal motor neurons. VFSS in these patients shows peculiar features, such as imperfect sealing of the posterior tongue due to muscle weakness or incoordination, which leads to leakage of $\mathrm{CM}$ in the oral pharynx before the beginning of the pharyngeal phase, or bolus aspiration in more severe cases $[10,11]$.

Tongue motility, assessed by evaluating the distance between the dorsum of the anterior tongue and hard palate, appears to be insufficient in ALS patients [10-12]. Defective tongue propulsion movement occurs as well and causes bolus stasis in the oral cavity or unsatisfactory repetitive backward bolus movements before swallowing [10-12].

In ALS patients defective sealing of the nasal-pharynx is often present, due to pharyngeal constrictor and soft palate muscle weakness, causing upward regurgitation of the bolus, or stasis in the valleculae and pyriform sinuses $[10,12]$.

VFSS can show also non-propulsive movement of the pharyngeal constrictor muscles, showing delayed progression of the bolus, which may cause episodes of post-deglutitive sub-epiglottic penetration. This feature is particularly dangerous in patients who have a defective cough reflex, or anomalies in swallowing-dependent apnoea (i.e. when swallowing apnoea is missed or, when it is present, it resumes with inspiration rather than expiration) $[3,6,10]$.

Laryngeal elevation delay time, defined as the time when the bolus head reaches the basal part of the pyriform recess and when the laryngeal elevation reaches its maximum, is often abnormal [10-12]. Insufficient or delayed elevation of the larynx and epiglottal tilting, often asymmetric (Figure 1), is a typical feature of ALS, especially in late stages of the disease, and leads to spillover of CM in the upper airway (in very critical patients it can even reach trachea) (Figure 2A) [10-12].

Deficitary propulsive contractions of oesophageal muscles, resulting in delayed progression or stasis, is also commonly reported (Figure $2 \mathrm{~B}$ ).

We should also mention that there is normally a good correlation between clinical severity class and the VFSS findings, but it has been reported that in ALS patients classified as having normal eating habits, clinical symptoms may be less severe compared to VFSS findings [13]. This may be due to underestimation of the initial symptoms, for which the patient tends to apply compensative manoeu- 
vres (adjusting postures, like head-flexed positions [Figure 2C]), that allow normal swallowing, although early muscle weakness or incoordination occur [10].

\section{Dementia}

Dementia is a neurodegenerative condition that encloses a broad category of brain diseases with similar features: fronto-temporal dementia (FTD), Alzheimer's disease (AD), dementia with Lewy bodies (DLB), and vascular dementia (VD) [13-15].

Dysphagia in these patients is not related to sensorymotor deficit but to a cognitive deterioration that causes an inability to recognise food, oral-tactile agnosia, disorders of chewing, swallowing, and feeding apraxia [13-15].

Even if VFSS can adequately assess swallowing impairment in a patient with dementia, the patient's cooperation is impaired, leading to difficulties in performing VFSS [14].

Although patients with dementia may have abnormal tongue and jaw movements, clinical examination cannot provide adequate information about the swallowing process, and VFSS becomes essential [16-18].

In patients with $\mathrm{VD}$, there is a greater involvement of the motor aspect of the swallowing, and a higher rate of silent aspiration than that encountered in patients affected by $\mathrm{AD}$.

FTD patients tend to show a lack of awareness of food in the mouth, which leads to rapid and compulsive eating of large amounts of food, and leaks into the pharynx are frequent [14]. Also, FTD is characterised by alterations in food preferences, appetite, and eating behaviour, which may be, as mentioned previously, worsened by a deficit of smooth muscle contractions and salivary gland secretion [14,16-19].

Swallowing alterations in DLB patients seems to be more related to pharyngeal phase anomaly, as well as the oral dysfunction both to apraxia and a reduced sensory input from the bolus [17].

Anomalies in oral stage swallowing are common in all types of dementia and may be present as functional disor-

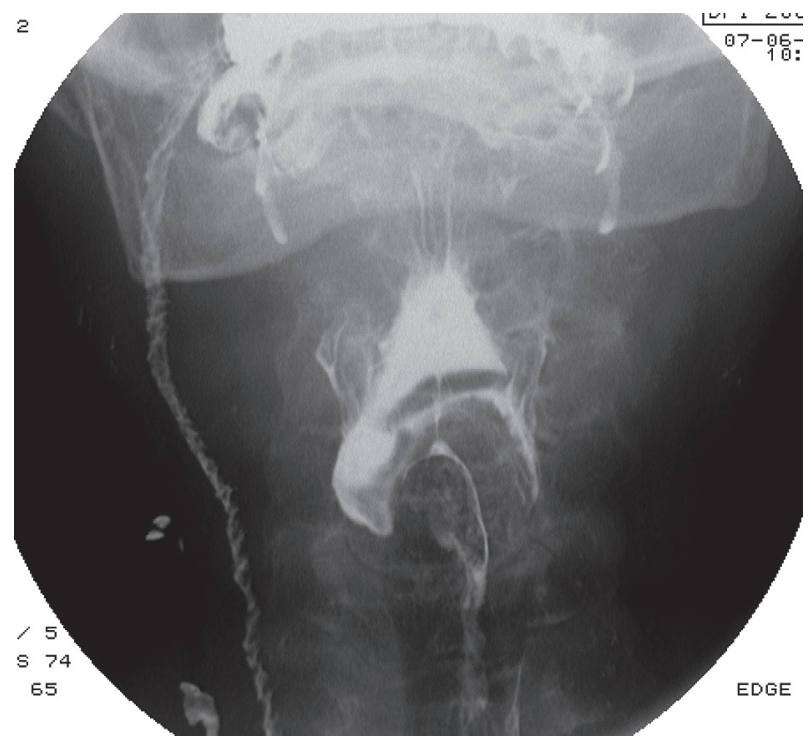

Figure 1. Videofluorographic swallowing study in the frontal view in a 75-year-old male with amyotrophic lateral sclerosis shows an asymmetry in epiglottal tilting and aspiration

ders like prolonged bolus preparation, pocketing food in the mouth, the need for reminders to swallow aliments, and disruption of the normal transition between oral and pharyngeal phase $[3,14,15,16,20]$.

Dysfunction of the autonomic nervous system, leading to reduced or absent salivary secretion and a deficit of smooth muscle contractions, may worsen these features $[15,20]$.

Moreover, in general, an insufficient amount of bolus ingested due to weakness or dyskinesia of tongue movement occurs as well, and this leads to delayed pharyngeal reflex and premature passage of a part of the bolus in the pharynx and laryngeal leaking. These features are more frequent when the patient swallows liquid bolus $[14,15,16-19,21]$. Incomplete bolus clearance, due to reduced capacity of contraction of the tongue, pharynx, and larynx, was also reported [14].

Anomalies in pharyngeal reflex and in airway protection mechanisms occur only in the late stage of the disease $[14,16,17]$. VFSS often shows delayed passage of the bolus

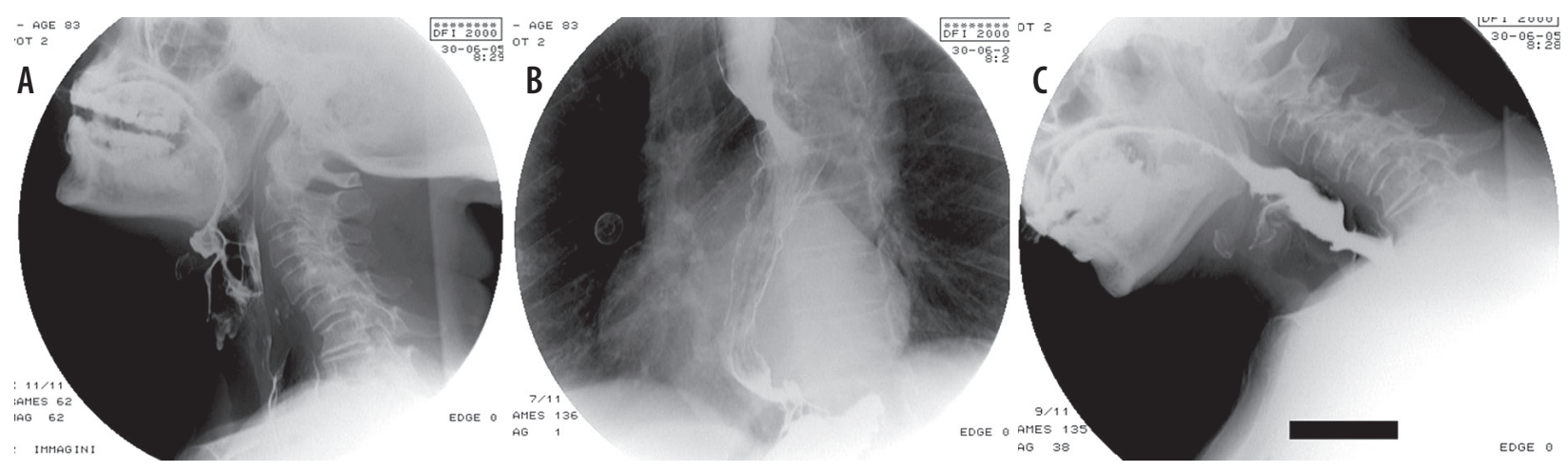

Figure 2. Videofluorographic swallowing study in the lateral view in an 83-year-old female with amyotrophic lateral sclerosis shows spillover of contrast media (CM) in the upper airway due to anomalies in epiglottal tilting and laryngeal elevation when the patient's head is extended (A). Oesophageal dyskinesia with non-propulsive tertiary waves (B); slight reduction of the spillover of $C M$ in the upper airway when the patient's head is flexed (C) 


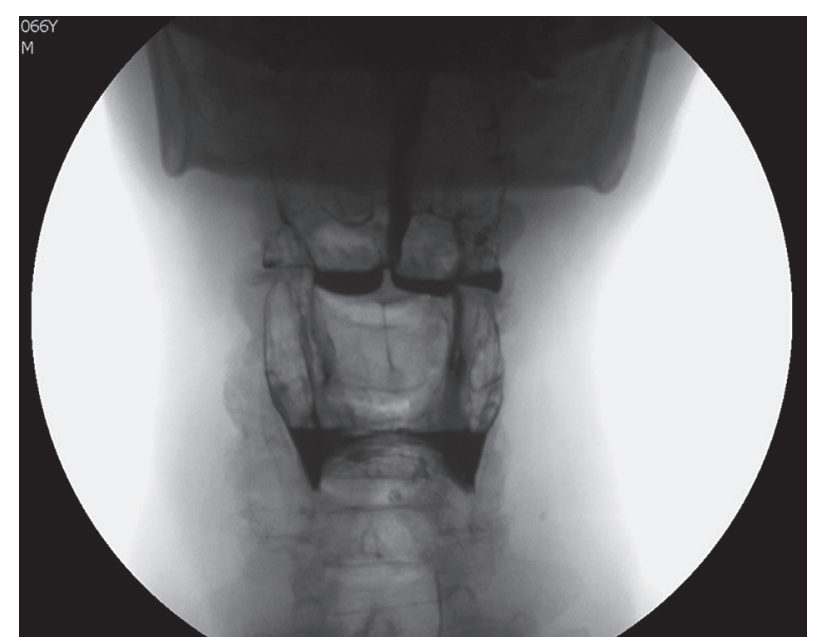

Figure 3. Videofluorographic swallowing study in the frontal view in a 66-year-old male with Alzheimer's disease shows retention of contrast media in valleculae and pyriform sinuses

in the pharynx and retention of CM in valleculae and/or pyriform sinuses (Figure 3) [21].

Pocketing of CM in the valleculae may be caused also by dysmotility of epiglottis [18]. This latter, together with reduced hypo-laryngeal excursion and incomplete sealing of the larynx, may be responsible of airway penetration or aspiration.

During VFSS, involuntary acquired movements must be assessed in order to guide nutritional management $[18,21,22]$. Minor penetration (entering the airway above the vocal folds) of CM may evoke immediate coughing when the airway protection mechanisms are active; in this case, artificial nutrition should not be suggested $[16,17,22]$.

\section{Multiple sclerosis}

Multiple sclerosis (MS) is the leading cause of neurological disability in young adults, that is why, especially in very young patients, like any radiological examination, VFSS must be performed with caution [23-26].

More than $30 \%$ of MS patients suffer from swallowing disorders, mainly in intermediate-advanced stages of the disease; the difficulty or inability to swallow is the result of the impairment of various neurological structures such as corticobulbar tract, brainstem, cerebellum, and cranial nerves $[27,28]$.

Neurogenic dysphagia in MS is associated with the degree of disability. It rarely appears in patients with mild disability and becomes more common with increasing disability [29], reaching a prevalence of $65 \%$ in severely disabled patients [29]. Although it is impossible to define the "typical" swallowing disorder patterns in MS patients, because this condition produces high variable motor performance impairment, we are going to describe the possible findings on VFSS.
VFSS reveals anomalies in oral and pharyngeal phases - characterised by tongue dysmotility and weakness, which leads to reduced bolus progression and multiple swallowing - and laryngeal dysmotility, a significantly shorter time frame from the onset of laryngeal excursion to return to rest, and longer time frame between airway closure and upper oesophageal sphincter opening [29,30]. This feature is particularly evident during liquid bolus swallowing, regardless of volume $[29,30]$. It is caused by the insufficient inactivation of the pharyngeal motor control of the pharynx by the brainstem neurons, so the airways remain open and the upper oesophageal sphincter remains closed, thus causing the massive passage of liquids in the pharynx [29].

The aforementioned reduced laryngeal elevation and weakened closure during swallowing can itself be the cause of the reduced pharyngeal clearance because laryngeal elevation contributes to the upper oesophageal sphincter opening $[31,32]$.

Other pharyngeal abnormalities may be present as well, due to delayed swallowing reflex, and progressive dysmotility and weakness of pharyngeal constrictor muscle, which are associated with the presence of $\mathrm{CM}$ residues in pyriform sinuses and valleculae [30-34].

It can be followed by episodes of post deglutitive penetration or aspiration, especially when associated with laryngeal and/or oesophageal dyskinesia [29].

Epiglottal dysmotility has been rarely reported [32]. Because the aforementioned SM has a higher incidence in young adults, close attention must be paid to radiation protection [35].

\section{Parkinson's disease}

Dysphagia in Parkinson's disease (PD) occurs in up to $50 \%$ of cases and is related to the rigidity and bradykinesia of the tongue and oropharyngeal muscles, associated with alterations in sensitive afferent integration, which guides the oral phase, even in early stages [36-40].

"Pumping" phenomenon is considered a pathognomonic feature of $\mathrm{PD}$, and it is caused by alterations of the labial sphincter closure and of the tongue movements [36-40]. Also, during chewing there is incoordination of tongue movement: the anterior portion performs repeated movements from the bottom upwards, while the posterior portion remains against the palate, thus preventing the progression of the bolus in the pharynx, resulting in post-swallowing oral CM residuals [36-40].

Drooling is one of the main features of dysphagia in PD patients, and it is caused by insufficient sealing of the labial sphincter, a reduction in the frequency of spontaneous swallowing, as well as an anteriorly flexed neck position (Figure 4) [40].

Pharyngeal phase anomalies are represented by the delayed swallowing reflex, reduced laryngeal elevation, and reduced strength of pharyngeal constrictor muscles, 


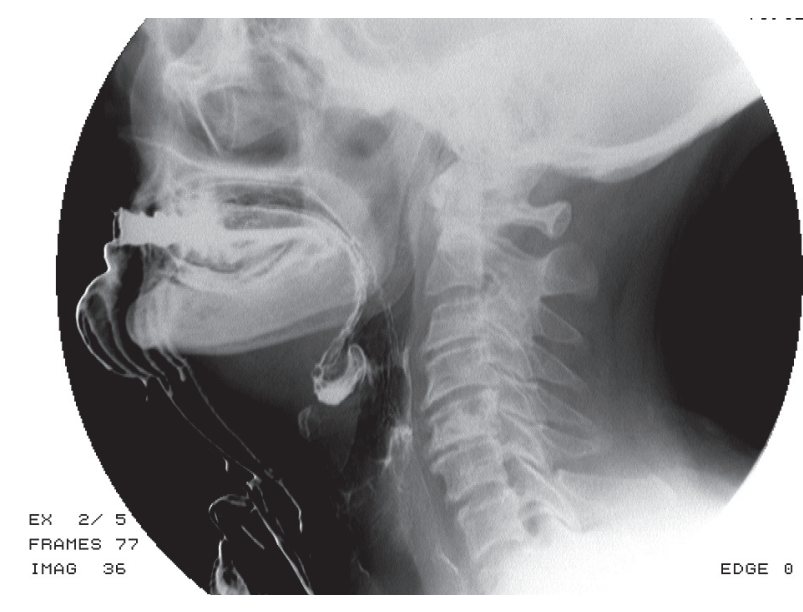

Figure 4. Videofluorographic swallowing study in the lateral view in a 71-year-old female with Parkinson's disease shows drooling and contrast media retention in the valleculae

leading to residue in the valleculae and pyriform sinuses and higher risk of aspiration [36-40].

This is a crucial issue for PD patients because coughing is frequently impaired and clearing the airway of foreign material becomes challenging. Patients with PD have also a decreased oesophageal peristalsis with VFSS appearance of tertiary waves, inefficient for bolus progression [37].

We should mention that in neurologic patients, not only in PD ones, the position of the head and the neck may affect swallowing: posture changing technique with "chin down" (flexion of the neck during swallowing) should be useful for PD patients with a history of suction [40,41].

It is important to notice that long-term treatment with levodopa, widely used in PD therapy, may lead to dyskinesia of the airway tract. This latter, associated with swallowing impairment, increases morbidity and mortality in advanced stages of PD [42].

\section{Huntington's disease}

Huntington's disease (HD)-related dysphagia is caused by choreiform movements of facial and neck muscles, together with tongue chorea, leading to inadequate tongue propulsion and mandibular rigidity, which results in intraoral bolus retention and/or segmented tongue transfer and repetitive swallowing, associated with food oral residue after swallowing solid food [43-45].

As mentioned before, swallowing abnormalities are associated with weakness and/or lack of coordination of the submental muscles, resulting in reduced hyoid bone and laryngeal elevation, which increases the risk of airway bolus leakage $[44,46,47]$. This is particularly dangerous when combined with the frequently reported inability to stop respiration during swallowing, and it leads to a higher risk of intra e post-deglutitive aspiration $[44,47]$.

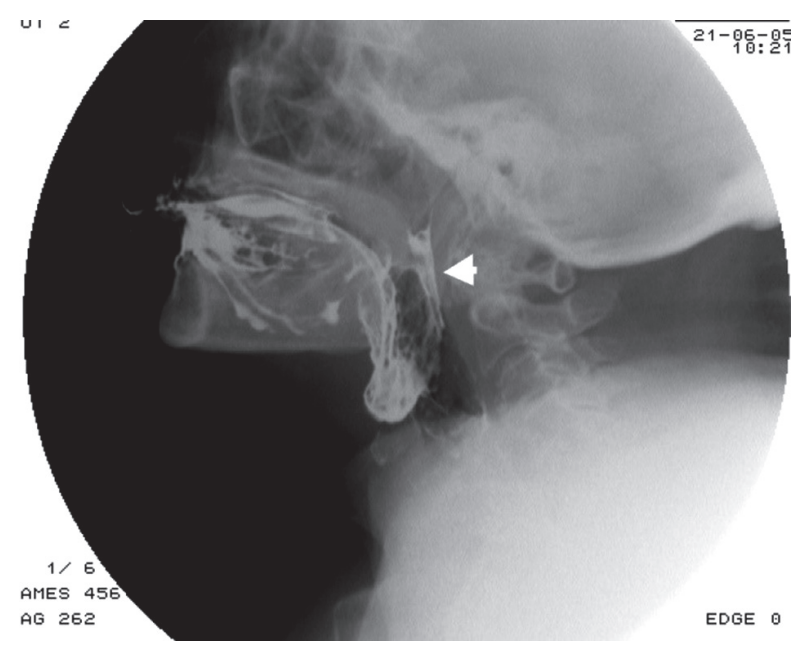

Figure 5. Videofluorographic swallowing study in the lateral view in a 70-year-old female with progressive supranuclear palsy shows contrast media reflux in rhino-pharynx (arrow)

\section{Progressive supranuclear palsy}

Progressive supranuclear palsy (PSP) is a neurodegenerative extrapyramidal disease in which swallowing disorders are common features; caused by pseudobulbar palsy, it main involve the oral phase. VFSS shows poor tongue-assisted mastication, uncoordinated tongue movements, and impaired posterior tongue displacement, along with the neck being held rigidly in extension [48,49]. The pharyngeal phase is less commonly affected. Absence of velar retraction or elevation, associated to posterior tongue displacement into the pharynx, vallecular CM bolus retention and copious pharyngeal secretions have been encountered [48,49] (Figure 5).

Despite the abnormal epiglottal movements, PSP patients tend to have lower aspiration risk than other neurodegenerative patients, also thanks to the hyperextension of the neck $[48,49]$.

Another peculiar feature of the swallowing mechanism in PSP is a type of pre-oral feeding pattern. Patients with PSP tend to overload the mouth with food or liquids, a pattern known as 'mouth stuffing and rapid drinking', so PSP patients have to be reminded 'not to overload the fork and mouth' $[48,49]$.

Oesophageal dysmotility may occur as well, leading to delayed bolus progression, tertiary contraction waves with bolus redirection, and LES impairment [49].

\section{Guillain-Barré syndrome, myasthenia gravis, and Lambert-Eaton syndrome}

Although they do not belong to the great chapter of neurodegenerative disorders, they deserve to be mentioned because they often complicate with dysphagia.

In Guillain-Barré syndrome (GBS), cranial nerves may be compromised, especially glossopharyngeal, vagus, and 


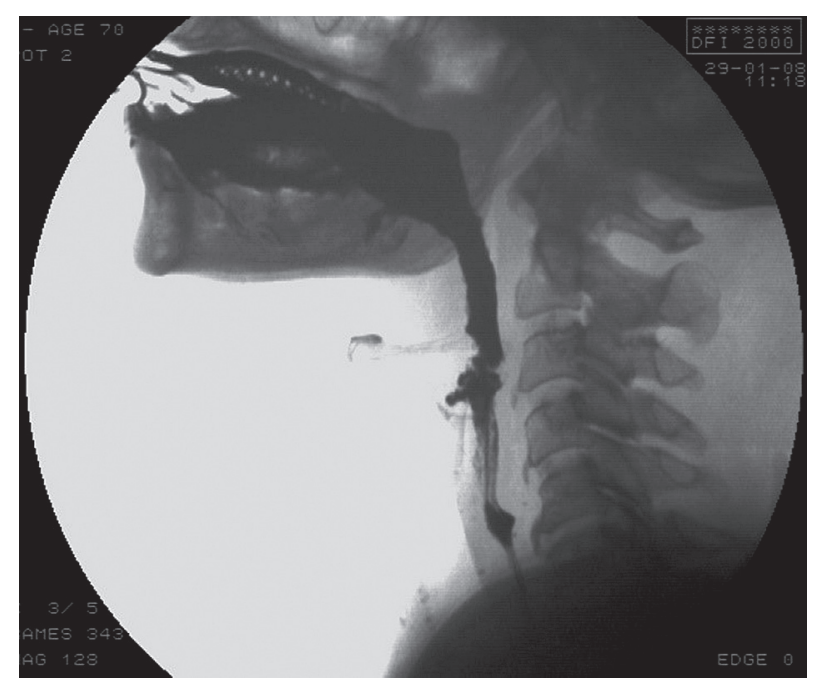

Figure 6. Videofluorographic swallowing study in the lateral view in a 70-year-old female with myasthenia gravis shows progressive weakness of palatal-pharyngeal muscles after a few swallows, associated with a delayed swallowing reflex and premature bolus pharyngeal leakage

facial nerves, leading to severe deterioration of the oropharyngeal phase [50].

MG and LE-S VFSS can show involvement of every phase of swallowing: jaw muscle weakness with impaired food trituration and bolus formation, soft palate incon- tinence and pharyngeal muscle impairment with bolus leakage in rhino-pharynx or slowed bolus progression through upper gastrointestinal tract [6,51].

Dynamic and repetitive swallowing show gradual decompensation (Figure 6), which characteristically improves after rest.

\section{Conclusions}

Swallowing impairment is one of the features that may be encountered in neurologic and neurodegenerative diseases. Its impact negatively affects patients' prognosis. Thus, its diagnosis and correct assessment with VFSS is useful and is needed to guide patient management. Neurologic diseases may have different patterns according to the impairment of the different phases of swallowing.

Radiologists should be familiar with the main VFSS features in these patients because early diagnosis of deglutition disorders widely improves patient outcome, and customising feeding (and overall therapy) strategies has a great impact both in "quoad-vitam" and "quoad-valetudinem" prognosis.

\section{Conflict of interest}

The authors report no conflict of interest.

\section{References}

1. Rugiu MG. Role of videofluoroscopy in evaluation of neurologic dysphagia. Acta Otorhinolaryngol Ital 2007; 27: 306-316.

2. Matsuo K, Palmer JB. Anatomy and physiology of feeding and swallowing - normal and abnormal. Phys Med Rehabil Clin N Am 2008; 19: 691-706.

3. Ott DJ, Pikna LA. Clinical and videofluoroscopic evaluation of swallowing disorders. AJR Am J Roentgenol 1993; 161: 507-513.

4. Scerrino G, Tudisca C, Bonventre S, et al. Swallowing disorders after thyroidectomy: What we know and where we are. A systematic review. Int J Surg 2017; 41 Suppl 1: S94-S102.

5. Dodds WJ, Stewart ET, Logemann JA. Physiology and radiology of the normal oral and pharyngeal phases of swallowing AJR Am J Roentgenol 1990; 154: 953-963.

6. Gore MR, Levine MS. Textbook of gastrointestinal radiology. Vol. 2. Saunders 2015.

7. Bonnie $\mathrm{MH}$, Bronwyn J. The videofluorographic swallowing study. Phys Med Rehabil Clin N Am 2008; 19: 769-785.

8. Russo S, Lo Re G, Galia M, et al. Videofluorography swallow study of patients with systemic sclerosis. Radiol Med 2009; 114: 948-959.

9. Lo Re G, De Luca R, Muscarneri F, et al. Relationship between anxiety level and radiological investigation. Comparison among different diagnostic imaging exams in a prospective single-center study. Radiologia Medica 2016; 121: 763-768.

10. Kawai S, Tsukuda M. Study of the early stage of dysphagia in amyotrophic lateral sclerosis. Dysphagia 2003; 18: 1-8.
11. Lo Re G, Galia M, La Grutta L, et al. Digital cineradiographic study of swallowing in patients with amyotrophic lateral sclerosis. Radiol Med 2007; 112: 1173-1187.

12. Leigh PN, Abrahams S, Al-Chalabi A, et al.; King's MND Care and Research Team. The management of motor neurone disease. J Neurol Neurosurg Psychiatry 2003; 74 (Suppl 4): iv32-iv47.

13. Salerno S, Lo Casto A, Romano I, et al. Morbidity of salivary gland digital sialography using a non-ionic dimeric contrast medium. Minerva Stomatol 2008; 57: 285-294.

14. Alagiakrishnan KA, Bhanji RA Kurian M. Evaluation and management of oropharyngeal dysphagia in different types of dementia: A systematic review. Arch Gerontol Geriatr 2013; 56: 1-9.

15. Affoo RH, Foley N, Rosenbek J, et al. Swallowing dysfunction and autonomic nervous system dysfunction in Alzheimer's disease: a scoping review of the evidence. J Am Geriatr Soc 2013; 61: 2203-2213.

16. Feinberg MJ, Knebl J, Tully J, Segall L. Aspiration and the elderly. Dysphagia 1990; 5: 61-71.

17. Ikeda M, Brown J, Holland AJ, et al. Changes in appetite, food preference, and eating habits in frontotemporal dementia and Alzheimer's disease. J Neurol Neurosurg Psychiatry 2002; 73: 371-376.

18. Londos E, Hanxsson O, Alm Hirsch I, et al. Dysphagia in Lewy body dementia - clinical observational study of swallowing function by videofluoroscopic examination. BMC Neurol 2013; 13: 140.

19. Salerno S, Cannizzaro F, Lo Casto A, et al. Interventional treatment of sialoliths in main salivary glands. Radiologia Medica 2002; 103 : 378-383. 
20. Priefer BA, Robbins J. Eating changes in mild-stage Alzheimer's disease: A pilot study. Dysphagia 1997; 12: 212-221.

21. Tracy JF, Logemann JA, Kahrilas PJ, et al. Preliminary observations on the effects of age on oropharyngeal deglutition. Dysphagia 1989; 4: 90-94.

22. Horner J, Alberts MJ, Dawson DV, Cook GM. Swallowing in Alzheimer's disease. Alzheimer Dis Assoc Disord 1994; 8: 177-189.

23. Colagrande S, Origgi D, Zatelli G, et al. CT exposure in adult and paediatric patients: A review of the mechanisms of damage, relative dose and consequent possible risks. Radiol Med 2014; 119: 803-810.

24. Granata C, Origgi D, Palorini F, et al. Radiation dose from multidetector CT studies in children: results from the first Italian nationwide survey. Pediatr Radiol 2015; 45: 695-705.

25. Salerno S, Marchese P, Magistrelli A, et al. Radiation risks knowledge in resident and fellow in paediatrics: A questionnaire survey. Ital J Pediatr 2015; 41: 21.

26. McFarlin DE, McFarland HF. Multiple sclerosis (first of two parts). N Engl J Med 1982; 307: 1183-1188.

27. McFarlin DE, McFarland HF. Multiple sclerosis (second of two parts). N Engl J Med 1982; 307: 1246-1251.

28. Solaro C, Rezzani C, Trabucco E, et al. Prevalence of patient-reported dysphagia in multiple sclerosis patients: An Italian multicenter study (using the DYMUS questionnaire). J Neurol Sci 2013; 331: 94-97.

29. Abraham SS, Yun PT. Laryngopharyngeal dysmotility in multiple sclerosis. Dysphagia 2002 Winter; 17: 69-74.

30. Logemann JA, Kahrilas PJ, Cheng J, et al. Closure mechanisms of laryngeal vestibule during swallow. Am J Physiol 1992; 262: G338-G344.

31. Rademaker AW, Pauloski BR, Colangelo LA, Logemann JA. Age and volume effects on liquid swallowing function in normal women. J Speech Hear Res 1998; 41: 275-284.

32. De Pauw A, Dejaeger E, D’hooghe B, Carton H. Dysphagia in multiple sclerosis. Clin Neurol Neurosurg 2002; 104: 345-351.

33. Hartelius L, Svensson P. Speech and swallowing symptoms associated with Parkinson's disease and multiple sclerosis: a survey. Folia Phoniatr Logop 1994; 46: 9-17.

34. Calcagno P, Ruoppolo G, Grasso MG, et al. Dysphagia in multiple sclerosis - prevalence and prognostic factors. Acta Neurol Scand 2002; 105: 40-43.

35. Palorini F, Origgi D, Granata C, et al. Adult exposures from MDCT including multiphase studies: First Italian nationwide survey. Eur Radiol 2014; 24: 469-483.
36. Nagaya M, Kachi T, Yamada T, Sumi Y. Videofluorographic observation on swallowing in patients with dysphagia due to neurodegenerative diseases. Nagoya J Med Sci 2004; 67: 17-23.

37. Logeman JA. Dysphagia in movement disorders. In: Jankovic J, Tolosa E (eds.). Advances in neurology. Vol. 49: Facial dyskinesias. Raven Press, New York 1988.

38. Robbin JA, Logemann JA, Kirshner HS. Swallowing and speech production in Parkinson's disease. Ann Neurol 1986; 19: 275-282.

39. Schneider JS, Diamond SG, Markham CH. Deficits in orofacial sensorimotor function in Parkinson' disease. Ann Neurol 1986; 19: 275-282.

40. Tjaden K. Speech and swallowing in Parkinson's disease. Top Geriatr Rehabil 2008; 24: 115-126.

41. Ekberg O. Posture of the head and pharyngeal swallowing. Acta Radiol Diagn 1986; 27: 691-696.

42. Monteiro L, Souza-Machado A, Pinho P, et al. Swallowing impairment and pulmonary dysfunction in Parkinson's disease: The silent threats 2010. J Neurol Sci 2014; 339: 149-152.

43. Sturrock A, Leavitt BR. The clinical and genetic features of Huntington disease. J Geriatr Psychiatry Neurol 2010; 23: 243-259.

44. Kagel MC, Leopold NA. Dysphagia in Huntington's disease: a 16year retrospective. Dysphagia 1992; 7: 106-114.

45. Hamakawa S, Koda C, Umeno H, et al. Oropharyngeal dysphagia in a case of Huntington's disease. Auris Nasus Larynx 2004; 31 : 171-176.

46. Kim J, Sapienza CM. Implications of expiratory muscle strength training for rehabilitation of the elderly: Tutorial. J Rehabil Res Dev 2005; 42: 211-224.

47. Kim Y, McCullough GH. Maximum hyoid displacement in normal swallowing. Dysphagia 2008; 42: 211-224

48. Rehman HU. Progressive supranuclear palsy. Postgrad Med J 2000; 76: 333-336

49. Johnston BT, Castell JA, Stumacher S, et al. Comparison of swallowing function in Parkinson's disease and progressive supranuclear palsy. Mov Disord 1997; 12: 322-327.

50. Lee KL, Lim OK, Lee JK, Park KD. Treatment of dysphagia with pyridostigmine bromide in a patient with the pharyngeal-cervical-brachial variant of Guillain-Barré syndrome. Ann Rehabil Med 2012; 36: 148-153.

51. Andrews PI. Autoimmune myasthenia gravis in childhood. Semin Neurol 2004; 24: 101 